\title{
Full Sheet Control Using Steerable Nips
}

\author{
Rene Sanchez, Member, IEEE, Roberto Horowitz, Senior Member, IEEE, and Masayoshi Tomizuka, Fellow, IEEE
}

\begin{abstract}
This paper describes the mechatronics design, prototype testing, and control of a steerable nips component for paper path mechanisms in high-speed color printers and photocopiers. When placed upstream from the image transfer station along the paper path, this device precisely controls the longitudinal, lateral, and skew directions of papers sheets, as they arrive to the image transfer station. The paper also presents a complete kinematic and dynamic analysis of the paper sheet steering mechanism, which is validated by experimental results. It is shown that the dynamics of a sheet under the control of the steerable nips mechanism are nonlinear and subject to nonholonomic constraints. A feedback linearization control strategy that includes dynamic surface control is developed and implemented to control the sheet's position and angular orientation under the condition that the sheet's speed in the longitudinal direction remains positive at all times. Experimental results verify that the steerable nips mechanism under the proposed feedback linearization control strategy is able to meet or exceed all design performance requirements for deployment as a component of an actual printer paper path control mechanism.
\end{abstract}

Index Terms-Nonholonomic, nonlinear control, paper sheet control, state feedback linearization.

\section{INTRODUCTION}

$\mathbf{S}$ TATE-OF-THE-ART paper path control requires the sheets to be accurately positioned as they arrive to the image transfer station. This includes correction for longitudinal, lateral, and angular errors. To accomplish this task, current machines have a registration station at the end of the paper path and before the image transfer station, which must correct for lateral and skew errors and deliver the sheet to the image transfer station on time and with a constant longitudinal velocity. However, most stateof-the-art registration devices cannot correct for errors at high speeds, whereas others mark the paper since they rely on high contact point forces to move the sheet laterally. In this paper, we present the mechatronics design, control, and experimental verification of an innovative device, known as the steerable nips mechanism [11] that permits a swifter correction of sheet lateral, longitudinal, and angular errors without marking the sheet. A prototype of this device was designed and built in the Department of Mechanical Engineering of the University of California at Berkeley (UCB). The sheet actuation mechanism in this device consists of two rollers, which are free to rotate parallel

Manuscript received March 25, 2008; revised December 27, 2008. First published March 27, 2009; current version published November 18, 2009. Recommended by Technical Editor M. Benbouzid. This work was supported in part by the National Science Foundation under Grant CMS 0301719 and in part by Xerox Corporation.

R. Sanchez is with Nikon Research Corporation of America, Belmont, CA 94002-4107 USA (e-mail: r2sanchez@gmail.com).

R. Horowitz and M. Tomizuka are with the Department of Mechanical Engineering, University of California at Berkeley, Berkeley, CA 94720-1740 USA (e-mail: horowitz@me.berkeley.edu; tomizuka@me.berkeley.edu).

Color versions of one or more of the figures in this paper are available online at http://ieeexplore.ieee.org.

Digital Object Identifier 10.1109/TMECH.2009.2015293 and perpendicular to the sheet, and will henceforth be referred to as "steerable nips." A freely rotating ball is spring-loaded against each of the steerable nips, providing a normal force that creates the friction force needed to propel the sheet through rolling, while minimizing the contact area between the steerable nip and sheet, hence decreasing the possibility of marking the sheet. By controlling the steering angle and spinning velocity of each of the two nips, it is possible to control the longitudinal position, lateral position, and angular orientation of the sheet in a somewhat analogous manner to steering a two-wheel robot; cf., [2] and [27]-[29]. However, unlike the two-wheel robot steering control problem, the sheet control under the steerable nips mechanism proposed in this paper has an additional degree of freedom, since the paper sheet can buckle, whereas the pavement does not. As will be explained in subsequent sections, the buckling of the paper makes it possible to linearize the system dynamics through feedback, whereas feedback linearization cannot be achieved in the two-wheel robot steering problem. The control system for the steerable nips mechanism must be designed so the paper is neither folded nor stretched too severely to prevent the paper from tearing or having skid marks from the nips.

Prior recent work in the area of sheet control in a printer paper path has primarily focused on the design of control architectures and control algorithms for coordinating multiple actuated sections of the paper path, in order to correct for longitudinal interspacing errors among sheets, and synchronize the arrival of a sheet to the image transfer station with its corresponding image, with respect to both time and longitudinal velocity [1], [3]-[6], [12]-[16], [18], [26]. However, none of these works address that issue of the simultaneous correction of paper sheet longitudinal, lateral, and angular positioning errors, which is the focus of this paper. Prior works that address this issue are more scarce, cf., [25], and is primarily in the form of patent disclosures. The description of the steerable nips mechanism in this paper, as well as its kinematic analysis, was first presented in [21]. However, because the fabrication of the steerable nips prototype was not completed, a simplified dynamic analysis of mechanism was only presented in [21], which did not include actuators' dynamics, as well as a significantly simplified version of the feedback control scheme presented in this paper and some simulation results. A hybrid automata-based control strategy for the model described in [21] is presented in [22], which has four finite states among which the control system switches during the trajectory tracking process. It is shown in [22] that by using a hybrid automata, it is possible to drive the paper from rest to any other position. A more complete dynamic model of the steerable nips mechanism in this paper is presented in [20], which included actuators' dynamics, as well as experimental results, some of which are included in this paper. Recently, a control strategy is presented in [7] for an underactuated steerable nips 
mechanism for printer sheet registration devices that is similar to the device presented in this paper. However, since the mechanism in [7] has one less degree of freedom than the mechanism presented in this paper and no sheet buckling nor actuators' dynamics is included in the control system design model, the control problem in [7] resembles more that of the steering a two-wheel robot, precluding the use of feedback linearization, and the resulting control strategy is significantly different from the one presented in this paper.

The objective of the steerable nips mechanism is to control the motion of a sheet of paper on an horizontal plane, from the time instant when the leading edge of the sheet enters in contact with the nips until the time instant when the trailing edge of the sheet losses contact with the nips, in order to reduce longitudinal, lateral, and angular position tracking errors to acceptable levels before the sheet enters the image registration station. The control strategy presented in this paper uses linearization by state feedback [23] and a backstepping technique similar to dynamic surface control [24], and considers a realistic steerable nip mechanism model that accounts for small amounts of sheet buckling and includes actuator dynamics. Simulation and experimental results are in close agreement and show that by using the proposed control strategy, the steerable nips mechanism can correct for large initial sheet tracking errors, which exceed industry specifications, and attain sheet tracking errors when the sheet enters the registration station that are well below industry requirements without damaging the sheet.

Section II describes in detail the design process of some of the important components of the experimental setup. In order to derive a realistic model of the controlled plant, the dynamic models for each of the actuators were derived and validated with experimental data, which is described in Section III. In Section IV, we derive the kinematic model, and in Section V, we derive the overall dynamic model of the steerable nips mechanism. The control strategy developed to control paper position and orientation of a sheet is presented in Section VI. The model and experimental results are presented in Section VII. Finally, conclusions and some comments regarding the design, control strategies, and experimental results are stated in Section VIII.

\section{EXPERIMENTAL SETUP}

The fixture shown in Fig. 1 was designed to allow the steerable nips concept shown in Fig. 2 to be tested. This mechanism has two rotating steerable rollers, which propel while steering a sheet in the horizontal plane. The fixture also has an exit roller, which moves the sheets out of the fixture as trailing edge of the papers losses contact with the steerable nips, and a feeder unit that feeds the sheets into the fixture.

The steerable nips mechanism in Fig. 2 can correct for lateral errors in the sheet's initial position, in addition to longitudinal and angular errors, without laterally moving the rollers, as is the case of most current registration and deskewing systems. This is achieved by steering the paper sheet through the coordinated angular rotation and steering of the two rollers. The rollers are designed to have only a point of contact with the sheet, as shown in Fig. 2. A ball is spring-loaded on top of each rollers,

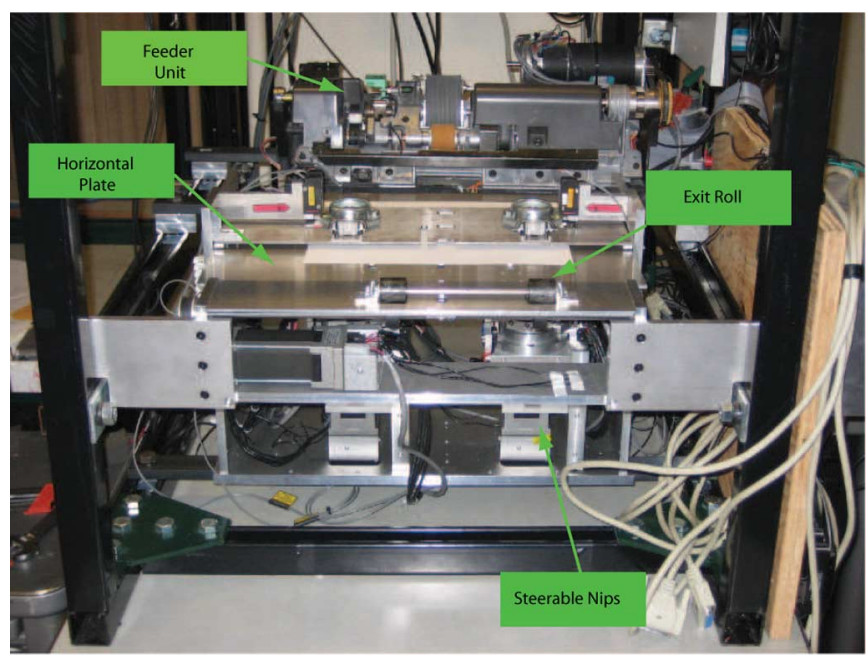

Fig. 1. Experimental setup.
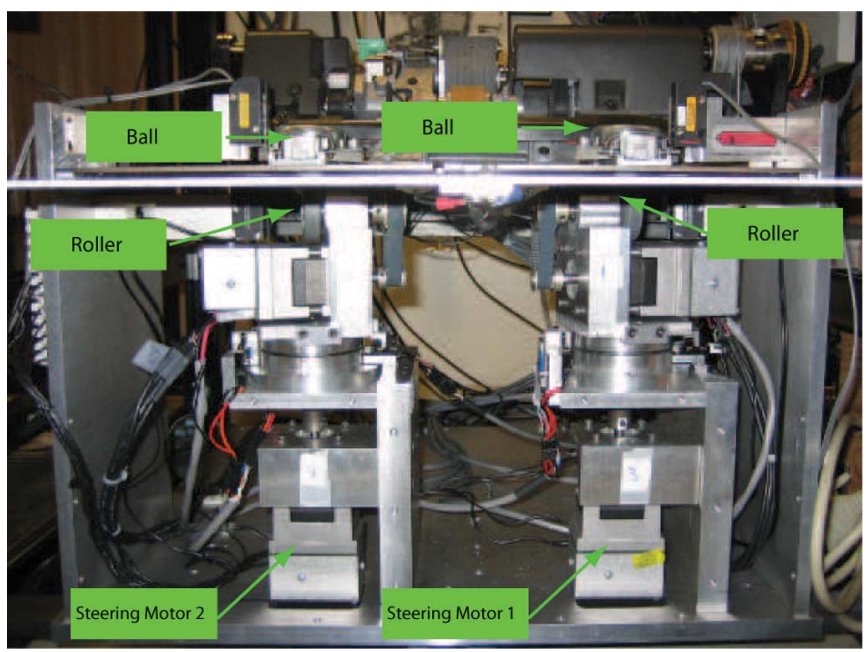

Fig. 2. Steerable nips mechanism.

providing a normal force that allows the rollers to drive the sheet. The combination of roller and ball is known as the nip. Properly controlled, the rollers can drive the sheet until its back edge exits the nips without inflicting any damage or visible skid marks on the sheet. Each roller is rotated by a servomotor, which will henceforth be referred to as the process motor, through a belt and pulleys, as shown in Fig. 2. The process motors were chosen to be light in weight and small in size in order to minimize the steering inertia. The two rollers are separated by a distance of $200 \mathrm{~mm}$. This spacing is compatible with standard sheet sizes. Each roller and its respective process motor are attached to a rotating table. The rotating table is steered by another servomotor, which will henceforth be referred to as the steering motor, as shown in Fig. 3. This steering table is connected to the servomotor through a precision shaft coupling. This coupling allows the rotating table to be driven directly by the servomotor under small shaft misalignment. This is a desirable feature since an additional belt would have decreased the stiffness of the steering mechanism. The rotating table sits on a trust bearing that allows it to freely rotate while supporting 


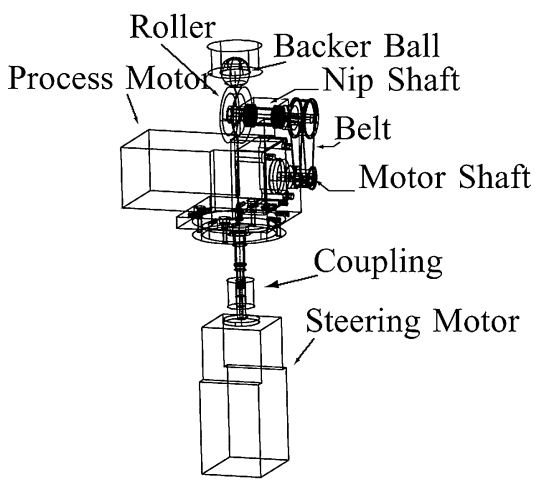

Fig. 3. Process direction actuator and steering motor mechanism.

TABLE I

PROCESS ACTUATOR VARIABLES AND EXPERIMENTALLY DETERMINED MODEL PARAMETERS

\begin{tabular}{|l|l|l|l|}
\hline Variable & Units & Description & Value \\
\hline $\mathrm{i}$ & Amps & Current & \\
$\theta_{m}$ & rad & Motor Ang. Pos. & \\
$\theta_{r}$ & rad & Roller Ang. Pos. & \\
$u$ & $V$ & Motor Input & \\
$K_{t}$ & $\frac{N-m}{A m p}$ & Motor Torque Cons. & 0.047 \\
$R$ & Ohms & Motor Res. & 19.8 \\
$L$ & $H e n$ & Motor Induc. & 0.0071 \\
$J_{m}$ & $N-m^{2}$ & Motor and Pulley Mo- & $3.1 \mathrm{e}-6$ \\
& & ments of Inertia & \\
$J_{r}$ & $N-m^{2}$ & Roller and Pulley Mo- & $1.95 \mathrm{e}-006$ \\
& & ments of Inertia & \\
$J_{b}$ & $N-m^{2}$ & Ball Moment of Inertia & $2.3 \mathrm{e}-7$ \\
$K$ & $\frac{N}{r a d}$ & Belt Spring Coef. & 369.2 \\
$B$ & $\frac{N s e c}{r a d}$ & Belt Damping Coef. & 1 \\
$B f_{m}$ & $\frac{N-m s}{r a d}$ & Motor Visc. Fric. & $6.5 \mathrm{e}-7$ \\
$B f_{r}$ & $\frac{N-m s}{r a d}$ & Roller Viscous Fric. & $9.55 \mathrm{e}-6$ \\
$B f_{b}$ & $\frac{N-m s}{r a d}$ & Ball Visc. Fric. & $3.50 \mathrm{e}-6$ \\
$r_{1}$ & $m$ & Motor Pulley Radius & 0.0058 \\
$r_{2}$ & $m$ & Roller Pulley Radius & 0.012 \\
$r_{3}$ & $m$ & Roller Radius & 0.02 \\
$r_{4}$ & $m$ & Ball Radius & 0.011 \\
\hline
\end{tabular}

its weight. There are also two ball bearings that prevent the rotating table from moving axially.

\section{ACTUATOR DYNAMICS}

There are four actuators in the steerable nips mechanism. Two of the actuators rotate each of the rollers and the other two steer them. In this section, we describe the dynamic model derivation, reduction, and validation for each of the actuators of the experimental fixture.

\section{A. Process Dynamics}

The process actuators rotate the two rollers and each consists of a dc servomotor, which drives a roller through a timing belt, as shown in Fig. 3. A fifth-order linear state-space model of this actuator was derived, which accounted for the stiffness of the timing belt and inductance of the dc motor. The parameters of the model were determined through experimental frequency response tests and are listed in Table I.

Fig. 4 plots the experimental frequency response of the transfer function between the voltage input and the roller angular
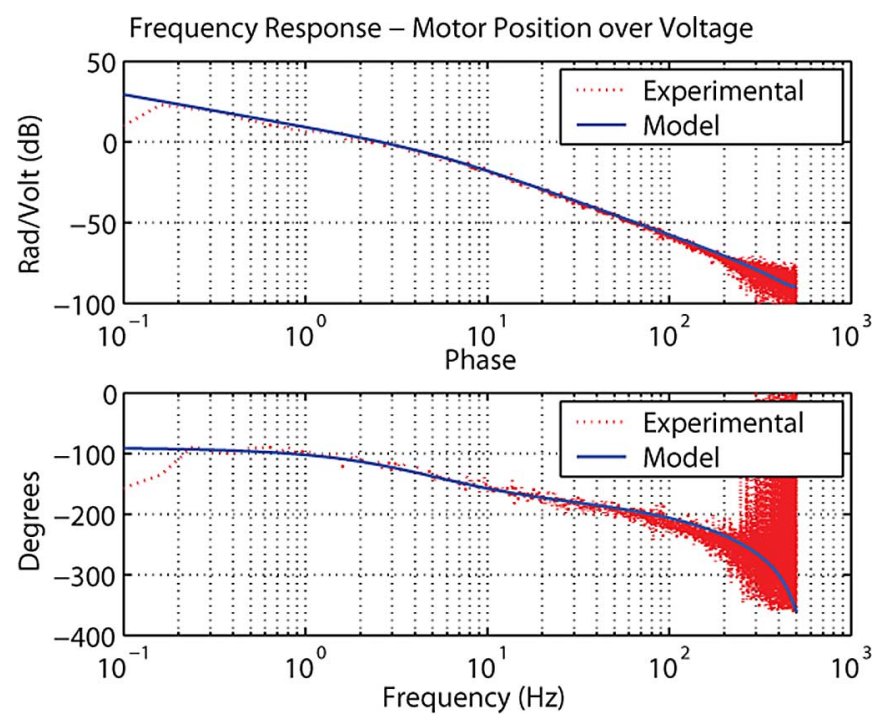

Fig. 4. Experimental and modeled frequency response of the process actuator.

rotation output of the process actuator, as well as the corresponding frequency response of the derived fifth-order model. As shown in the figure, the model frequency response closely matches experimental data at frequencies below $100 \mathrm{~Hz}$.

The fifth-order process actuator model was reduced to a second-order model by neglecting the inductance of the dc motor and assuming that the timing belt connecting the two pulleys of the actuator is very stiff. The resulting transfer function between the input voltage and the roller angular rotation output is

$$
\ddot{\theta}_{r}+\alpha_{p} \dot{\theta}_{r}=\beta_{p} u
$$

where

$$
\alpha_{p}=\frac{B f_{r}+\left(r_{3}^{2} / r_{4}^{2}\right) B f_{b}+\left(B f_{m}+\left(K_{t}^{2} / R\right)\right)\left(r_{2}^{2} / r_{1}^{2}\right)}{J_{r}+\left(r_{3}^{2} / r_{4}^{2}\right) J_{b}+\left(r_{2}^{2} / r_{1}^{2}\right) J_{m}}
$$

and

$$
\beta_{p}=\frac{\left(r_{1} / r_{2}\right)\left(K_{t} / R\right)}{J_{r}+\left(r_{3}^{2} / r_{4}^{2}\right) J_{b}+\left(r_{2}^{2} / r_{1}^{2}\right) J_{m}} .
$$

The frequency response of the simplified model (1) can be seen in Fig. 5. It is shown that the magnitude of the second-order model closely matches the fifth-order model in the frequency range shown. As expected, the phase of the reduced-order model diverges from the fifth-order model at high frequencies.

\section{B. Steering Dynamics}

The steering actuator consists of a dc servomotor that rotates a table, as shown in Fig. 3. This table holds the process actuator and is connected to the motor through a coupling. A third-order linear state-space model of this actuator was derived, which accounted for inductance of the dc motor. The parameters of the model were determined through experimental frequency response tests and are listed in Table II.

Fig. 6 plots the experimental frequency response of the transfer function between the voltage input and the steering angular rotation, as well as the corresponding frequency response of the 

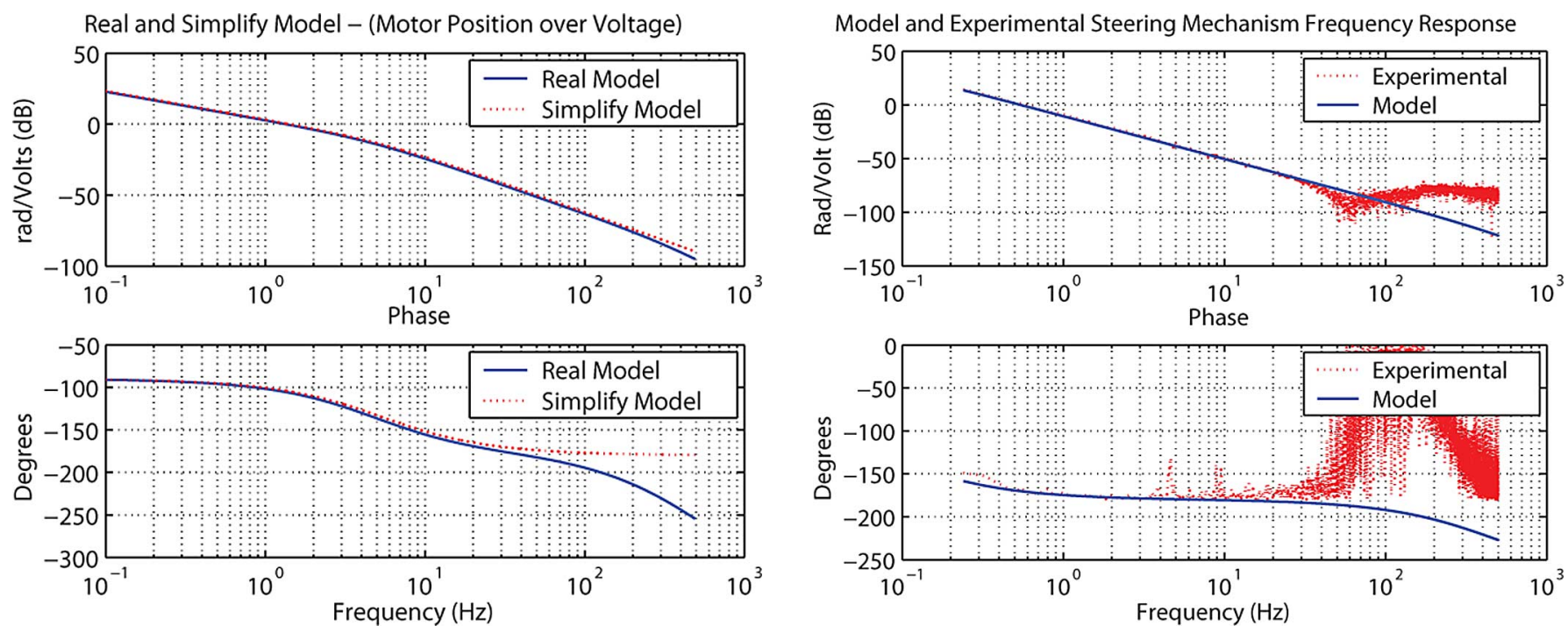

Fig. 5. Frequency responses of the process motor high-order and reducedorder models.

TABLE II

STEERING ACTUATOR VARIABLES AND EXPERIMENTALLY DETERMINED PARAMETER VALUES

\begin{tabular}{|l|l|l|l|}
\hline Variable & Units & Description & Value \\
\hline $\mathrm{i}$ & Amps & Current & \\
$\phi$ & $r a d$ & Steering Ang. & \\
$u$ & $V$ & Motor Input & \\
$K_{t}$ & $\frac{N-m}{A m p}$ & Motor Torque Cons. & 0.05 \\
$R$ & Ohms & Motor Res. & 6.78 \\
$L$ & $H e n$ & Motor Ind. & 0.0023 \\
$J_{t}$ & $N-m^{2}$ & Total Moment of Inertia & $6.24 \mathrm{e}-4$ \\
$B f_{m}$ & $\frac{N}{r a d}$ & Motor Visc. Fric. & $1.4 \mathrm{e}-6$ \\
\hline
\end{tabular}

derived third-order model. As shown in the figure, the model frequency response closely matches experimental data at frequencies below $40 \mathrm{~Hz}$.

The mismatch between the modeled and experimental results for frequencies higher than $40 \mathrm{~Hz}$ can be attributed to low signal-to-noise ratio. The system was exited with white noise and the excitation level did not produce a sufficiently large displacement at high frequency to overcome the encoder accuracy. The estimated values for the parameters of the steering actuators are also shown in Table II. As in the case of the process actuators, the dynamic model of the steering mechanism can be reduced by assuming that the inductance of the motor is negligible $(L(d i / d t) \approx 0)$. The resulting second-order transfer function is given by

$$
\ddot{\phi}+\alpha_{s} \dot{\phi}=\beta_{s} u
$$

where

$$
\alpha_{s}=\frac{\left(B f+\left(K_{t}^{2} / R\right)\right)}{J_{t}} \text { and } \beta_{s}=\frac{K_{t}}{J_{t} R}
$$

The frequency responses of the second-order and third-order models are shown in Fig. 7.

Fig. 6. Experimental and modeled steering actuator frequency response.
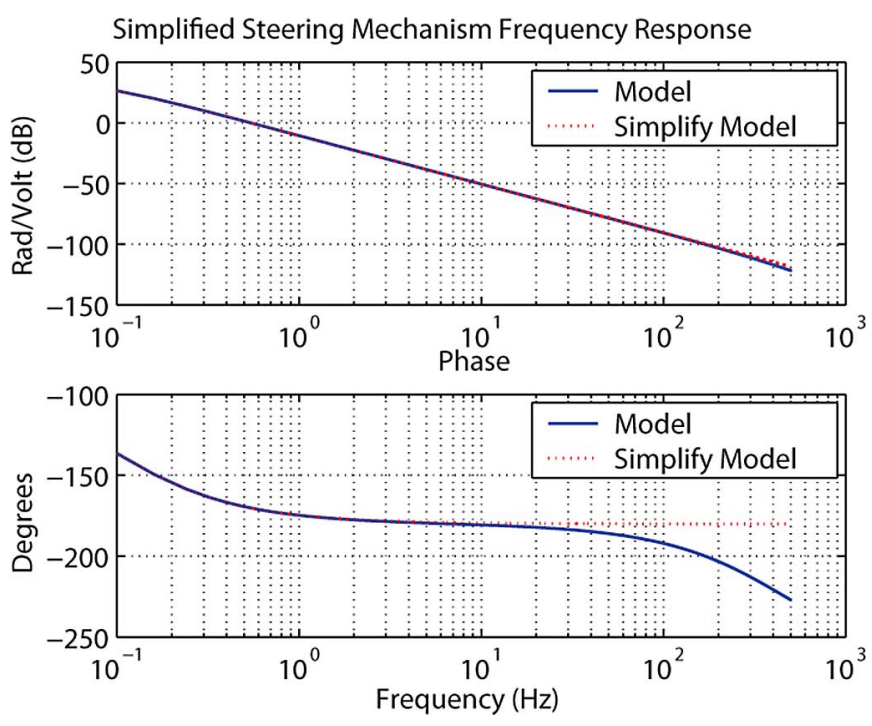

Fig. 7. High-order and reduced-order steering actuator frequency response.

\section{KinematiC ANALYSIS}

The steerable nips mechanism with a sheet is illustrated in Figs. 8 and 9. The steerable nips propel a sheet on a flat surface. Fig. 8 represents the initial position of the sheet, when it first makes contact with the nips. Fig. 9 shows the sheet while it is being driven by the nips. The left corner of the sheet, point $C$, is used as a reference point for tracking. Note that even if the paper buckles, point $C$ will remain on the flat surface since bucking occurs only between points 1 and 2 where the sheet contacts the nips.

\section{A. Kinematic Analysis Assumption}

A key modeling assumption in the kinematic analysis that follows is that, even under buckling, the sheet remains transversally stiff and it rotates as a rigid body under the action of the 


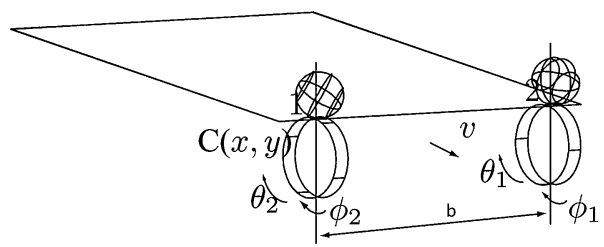

Fig. 8. Sheet entering the steerable nips system.

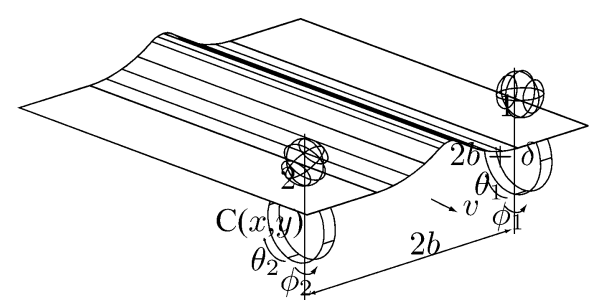

Fig. 9. Steerable nips with sheet buckle.

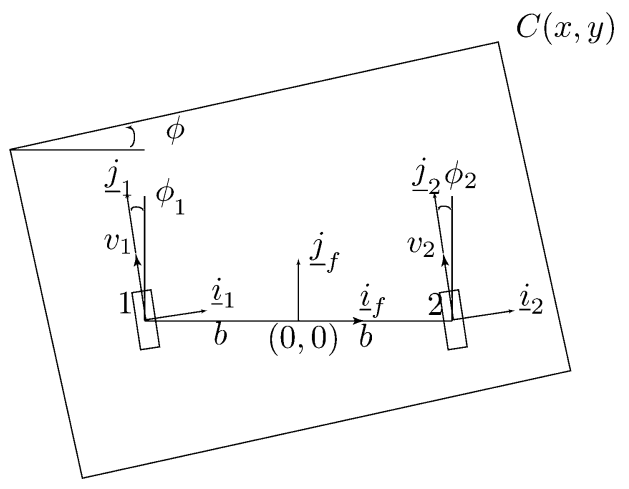

Fig. 10. System coordinates.

steerable nips. This assumption is illustrated in Fig. 9, where any line perpendicular to the line that connects contact points 1 and 2 drawn along the buckled surface remains horizontal. Numerous experimental results confirmed the validity of this assumption to within possible measurement resolution.

\section{B. Notation}

Fig. 10 shows a schematic representation of the kinematic variables for the steerable nips system. This system has two independent steering wheels located at points 1 and 2 . These steerable wheels are separated by a distance $2 b$. Three coordinate frames are defined to describe the position and orientation of the paper sheet: a fixed global coordinate system denoted by $\left(\underline{i}_{f}, \underline{j}_{f}, \underline{k}_{f}\right)$, and two local frames $\left(\underline{i}_{1}, \underline{j}_{1}, \underline{k}_{1}\right)$ and $\left(\underline{i}_{2}, \underline{j}_{2}, \underline{k}_{2}\right)$ attached to wheels 1 and 2, respectively. The generalized coordinates of the system are $\left(x, y, \phi, \delta, \theta_{1}, \theta_{2}, \phi_{1}, \phi_{2}\right)$. Generalized coordinates $x$ and $y$ will be used to represent the lateral and longitudinal position of the leading right corner of the sheet (point $C$ ), respectively. Coordinate $\phi$ represents the angular (or skew) rotation of the sheet and $-\delta$ represents the amount of buckle experienced by the sheet, which we assume to only take place along the line that connects points 1 and 2 . The angular positions of wheel 1 in the directions parallel and perpendicular to the sheet are denoted as $\theta_{1}$ and $\phi_{1}$, respectively. Likewise, $\theta_{2}$ and $\phi_{2}$ describe the angular position of wheel 2 parallel and perpendicular to the sheet.

\section{Velocity Analysis}

The velocities of the paper at points 1 and 2 in global coordinates are

$$
\begin{aligned}
& \underline{v}_{1}=(\dot{x}+\dot{\phi} y) \underline{i}_{f}+(\dot{y}-\dot{\phi}(x+b)) \underline{j}_{f}-\dot{\delta} \underline{i}_{f} \\
& \underline{v}_{2}=(\dot{x}+\dot{\phi} y) \underline{i}_{f}+(\dot{y}+\dot{\phi}(-x+b)) \underline{j}_{f}
\end{aligned}
$$

where $\dot{\delta}$ is the rate of change of the sheet buckle (or stretch), which is assumed to only take place along the direction of the line that connects point 1 and 2 (along the $\underline{i}_{f}$ unit vector). Note that a negative $\delta$ implies that the sheet has buckled while a positive $\delta$ implies that the sheet has stretched. Invoking the nonslip condition, $\underline{v}_{1}$ and $\underline{v}_{2}$ can also be written in terms of the angular speed of the wheels in the local coordinate frames

$$
\begin{aligned}
& \underline{v}_{1}=-r_{1} \dot{\theta}_{1} \underline{j}_{1} \\
& \underline{v}_{2}=-r_{2} \dot{\theta}_{2} \underline{j}_{2}
\end{aligned}
$$

where $r_{1}$ and $r_{2}$ are the radius of wheels 1 and 2, respectively.

Four constraint equations are obtained by writing (5) and (6) in terms of the global coordinates and equating them to (3) and (4)

$$
\begin{aligned}
\underline{v}_{1} & =-r_{1} \dot{\theta}_{1}\left[-\sin \phi_{1} \underline{i}_{f}+\cos \phi_{1} \underline{j}_{f}\right] \\
& =(\dot{x}+\dot{\phi} y-\dot{\delta}) \underline{i}_{f}+(\dot{y}-\dot{\phi}(x+b)) \underline{j}_{f} \\
\underline{v}_{2} & =-r_{2} \dot{\theta}_{2}\left[-\sin \phi_{2} \underline{i}_{f}+\cos \phi_{2} \underline{j}_{f}\right] \\
& =(\dot{x}+\dot{\phi} y) \underline{i}_{f}+(\dot{y}-\dot{\phi}(x-b)) \underline{j}_{f} .
\end{aligned}
$$

These equations result in four nonholonomic constraints

$$
\begin{aligned}
\dot{x}+\dot{\phi} y-\dot{\delta} & =r_{1} \sin \phi_{1} \dot{\theta}_{1} \\
\dot{y}-\dot{\phi}(x+b) & =-r_{1} \cos \phi_{1} \dot{\theta}_{1} \\
\dot{x}+\dot{\phi} y & =r_{2} \sin \phi_{2} \dot{\theta}_{2} \\
\dot{y}-\dot{\phi}(x-b) & =-r_{2} \cos \phi_{2} \dot{\theta}_{1} .
\end{aligned}
$$

Defining the generalized coordinate $p=\left[\begin{array}{llllll}x & y & \phi & \delta & \theta_{1} & \theta_{2}\end{array}\right.$ $\left.\phi_{1} \phi_{2}\right]^{T}$, the constraints can be written in Pfaffian form [17] as

$$
A(p) \dot{p}=0
$$

where $A(p)$ is the $4 \times 8$ matrix defined as

$$
\begin{aligned}
& A(p) \\
& \quad=\left[\begin{array}{cccccccc}
1 & 0 & y & -1 & -r_{1} \sin \phi_{1} & 0 & 0 & 0 \\
0 & 1 & -(x+b) & 0 & r_{1} \cos \phi_{1} & 0 & 0 & 0 \\
1 & 0 & y & 0 & 0 & -r_{2} \sin \phi_{2} & 0 & 0 \\
0 & 1 & -(x-b) & 0 & 0 & r_{2} \cos \phi_{2} & 0 & 0
\end{array}\right] .
\end{aligned}
$$




\section{Kinematic Equations}

The kinematic model represents the relation between the moving parts of the system, when they comply with the nonholonomic constraints describe earlier. As detailed in [17], this is given by a basis of the right null space of the constraints $a_{i}(p)$, which will be denoted by $g_{j}(p) \in \mathbb{R}^{n}, j=1, \ldots, n-k=m$. By construction, this basis satisfies

$a_{i}(p) g_{j}(p)=0, \quad i=1, \ldots, k, \quad j=1, \ldots, n-k, \quad p \in \mathbb{R}^{n}$ and all allowable trajectories of the system can thus be written as the possible solutions of the system

$$
\dot{p}=g_{1}(p) u_{1}+\cdots+g_{m}(p) u_{m}
$$

i.e., $p(t)$ is a feasible trajectory of the system if and only if $p(t)$ satisfies (14) for a choice of control $u(t) \in \mathbb{R}^{m}$.

For our system, this basis is easily obtained and the solution can be written as follows:

$\dot{x}=-\frac{y r_{1}}{2 b} \cos \phi_{1} \dot{\theta}_{1}+\left(\frac{y r_{2}}{2 b} \cos \phi_{2}+r_{2} \sin \phi_{2}\right) \dot{\theta}_{2}$

$\dot{y}=\left(\frac{(x+b) r_{1}}{2 b} \cos \phi_{1}-r_{1} \cos \phi_{1}\right) \dot{\theta}_{1}-\frac{(x+b) r_{2}}{2 b} \cos \phi_{2} \dot{\theta}_{2}$

$\dot{\phi}=\frac{1}{2 b}\left(r_{1} \cos \phi_{1} \dot{\theta}_{1}-r_{2} \cos \phi_{2} \dot{\theta}_{2}\right)$

$\dot{\delta}=r_{2} \sin \phi_{2} \dot{\theta}_{2}-r_{1} \sin \phi_{1} \dot{\theta}_{1}$

which constitute the kinematic model of our system. Note that (15)-(18) can be succinctly written as follows:

$$
\underline{y}=G(\underline{y}, \eta) \eta
$$

where the output

$$
\underline{y}=\left[\begin{array}{llll}
x & y & \phi & \delta
\end{array}\right]^{T}
$$

is the generalized coordinate vector of the paper sheet, with elements given, respectively, by the lateral and longitudinal coordinates $x$ and $y$ for the sheet's leading edge right corner $C$ (see Fig. 10), the sheet angular rotation $\phi$, and the buckle $\delta$, and

$$
\eta=\left[\begin{array}{llll}
\dot{\theta}_{1} & \dot{\theta}_{2} & \phi_{1} & \phi_{2}
\end{array}\right]^{T}
$$

is the input vector formed by the nips' rotor angular speeds and steering angles.

\section{E. Sheet Motion Sensing and Kinematic Model Experimental Validation}

An array of optical sensors, located along the longitudinal direction in the midpoint between the two rollers, as shown in Fig. 11, was used to detect the leading edge of the sheet. Three laser sensors, two of which are located on the right side of the sheet at locations $\left(\bar{x},-d_{s}\right)$ and $(\bar{x}, 0)$, respectively, and one is located on the left size of the sheet at location $(-\bar{x}, 0)$, as shown in Fig. 11, were used to measure the lateral deviation of the sheet edge relative to the sensor location (e.g., $s_{1}$ and $s_{2}$ are zero if the right-hand sheet edge is on top of both sensors). The amount of buckle $-\delta$ was determined from the readings $s_{2}$ and $s_{3}$ of the two laser sensors located on opposite sides of the sheet,

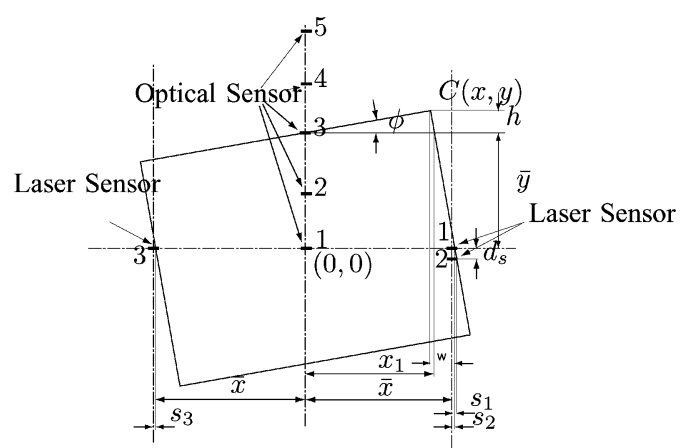

Fig. 11. Sheet position from optical and laser sensors.

while the angular position of the sheet $\phi$ was determined from readings $s_{1}$ and $s_{2}$ of the two laser sensors on the right side of the sheet. They are, respectively, given by

$$
\delta=s_{2}-s_{3} \quad \phi=\tan ^{-1}\left(\frac{s_{1}-s_{2}}{d_{s}}\right)
$$

where $d_{s}$ is the longitudinal distance between the two laser sensors 1 and 2, as shown in Fig. 11.

The position of point $C(x, y)$ on the right side of the leading edge of the sheet was determined every time the leading edge of the sheet went over one of the optical sensors. The longitudinal position of each sensor that is being crossed is denoted by $\bar{y}$, then the coordinates $y$ and $x$ of the point $C(x, y)$ are, respectively, given by

$$
\begin{aligned}
& y=\bar{y}+\left(\bar{x}-\bar{y} \tan \phi+s_{2}\right) \sin \phi \cos \phi \\
& x=\bar{x}-y \tan \phi
\end{aligned}
$$

where $2 \bar{x}$ is the lateral distance between laser sensors 2 and 3 (see Fig. 11). The kinematic state model equations given by (19) were validated using the following procedure: first, a sheet of paper was fed into and controlled by the steerable nips mechanism until it exited, while all sensor data were collected and stored. Second, the initial generalized coordinate vector of the paper sheet $y(0)$ was set to the values either directly measured or computed from (20) and (21) at the instant when the leading edge of the sheet went over the first optical longitudinal sensor. Subsequently, (19) were numerically integrated using as inputs the measured roller angular speed and steering angle of each nip. Finally, the numerically integrated sheet longitudinal, lateral, and angular positions $[y(t), x(t), \phi(t)]$ were plotted along with actual values computed from (20) and (21) at the instances when the leading edge of the sheet went over one of the optical longitudinal sensors. Typical results are shown in Figs. 12-14.

Note that there is a close match between the measured and predicted data in spite of the fact that the edges of paper sheets are not perfectly straight, and the data were contaminated by sensor and quantization noise. The angular velocities and steering angles used for the experimental validation of the kinematic equations were chosen in order not to induce appreciable buckle. Whatever buckle values were obtained from measurements and (20) were within the range of error attributed to the fact that the sheets are not all exactly of the same length and their edges are not parallel to each other. 


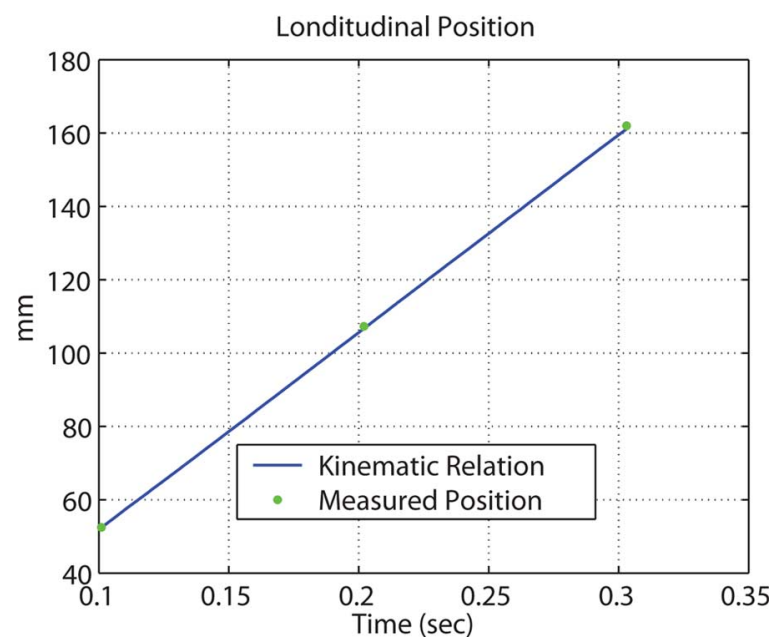

Fig. 12. Predicted and measured sheet longitudinal position.

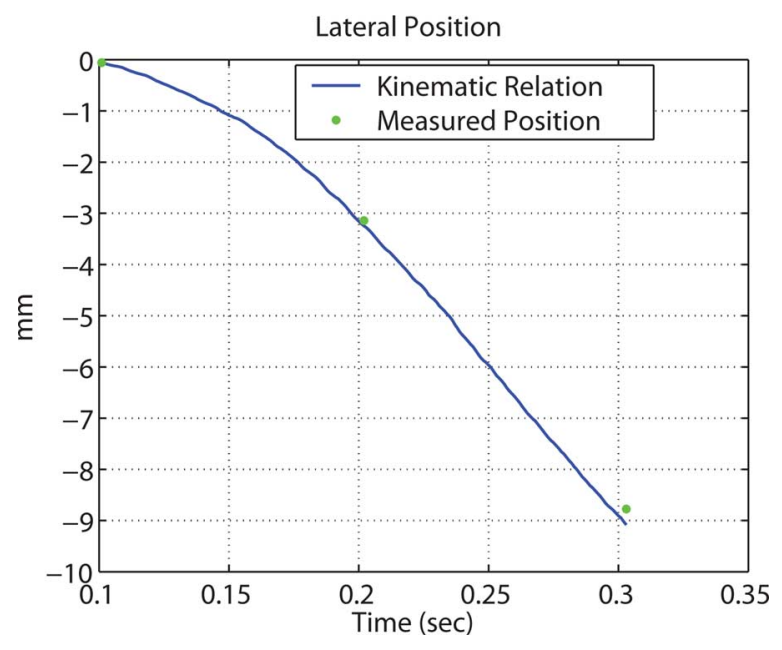

Fig. 13. Predicted and measured lateral sheet position.

\section{DYNAMIC MODEL}

\section{A. Dynamic Analysis Assumptions}

The key modeling assumption used in the dynamic analysis that follows is that the mass of the sheet is small, and therefore, it can be neglected. As a consequence, the overall model of the steerable mechanism consists of the dynamics of the actuators and the kinematic relation between the roller and the sheet. We also assume that a minute stretching of the sheet will not introduce dynamic coupling between the process and steering motors. These assumptions were also verified with numerous experimental results.

\section{B. Dynamic System With Reduced-Order Servo Dynamics}

In this section, we derived a model based on the actuator dynamics presented in Sections III-A and III-B. These models have the following simplified form:

$$
\ddot{\theta}_{i}+\alpha_{i} \dot{\theta}_{i}=\beta_{i} u_{i}
$$

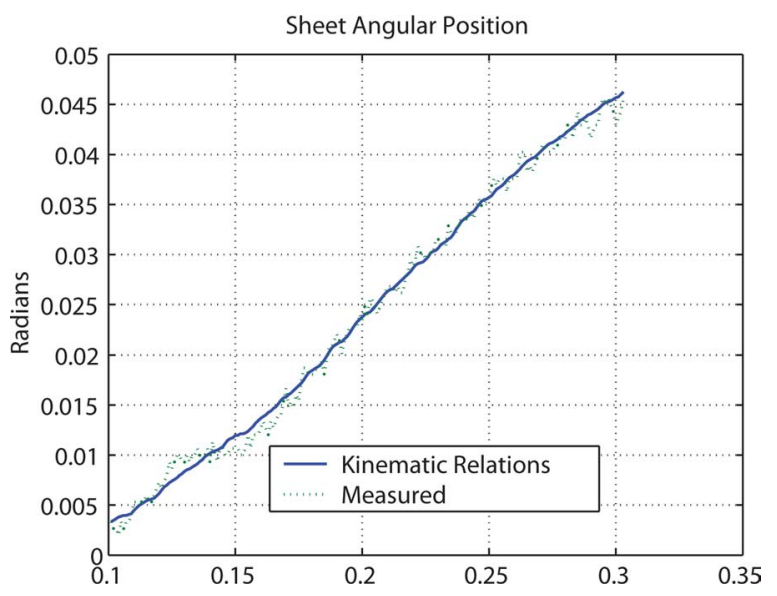

Fig. 14. Predicted and measured sheet angular position.

where $\alpha_{i}$ and $\beta_{i}(i=1,2,3,4)$ represent physical characteristics of each actuator defined in (1) and (2). Inputs $u_{1}, u_{2}, u_{3}$, and $u_{4}$ are motor voltages to each of the servomotors. Making use of the kinematic equations derived in Section IV-D, (19) and its time derivative can be expressed as follows:

$$
\begin{aligned}
& \underline{\ddot{y}}=M(\underline{x})+N(\underline{x})\left[\begin{array}{c}
\ddot{\theta}_{1} \\
\ddot{\theta}_{2} \\
\dot{\phi}_{1} \\
\dot{\phi}_{2}
\end{array}\right] \\
& \underline{y}=\left[\begin{array}{llll}
x & y & \phi & \delta
\end{array}\right]^{T} \\
& \underline{x}=\left[\begin{array}{llllllllll}
x & y & \phi & \delta & \phi_{1} & \phi_{2} & \dot{\theta}_{1} & \dot{\theta}_{2} & \dot{\phi}_{1} & \dot{\phi}_{2}
\end{array}\right]^{T}
\end{aligned}
$$

where $y$ is the output vector, and the vector $M(\underline{x}) \in \mathcal{R}^{3}$ is given by

$$
M(\underline{x})=\left[\begin{array}{c}
-\dot{\phi} \dot{y} \\
\dot{\phi} \dot{x} \\
0 \\
0
\end{array}\right]
$$

where the symbols $\dot{x}, \dot{y}, \dot{\phi}$, and $\dot{\delta}$ should be, respectively, replaced by the right-hand side of (15)-(18), and the elements of the matrix $N(\underline{x}) \in \mathcal{R}^{3 \times 3}$ are

$$
\begin{aligned}
& n_{11}=-\frac{y r_{1}}{2 b} \cos \phi_{1} \quad n_{12}=\frac{y r_{2}}{2 b} \cos \phi_{2}+r_{2} \sin \phi_{2} \\
& n_{13}=\frac{y r_{1}}{2 b} \sin \phi_{1} \dot{\theta}_{1} \quad n_{14}=-\frac{y r_{2}}{2 b} \sin \phi_{2} \dot{\theta}_{2}+r_{2} \cos \phi_{2} \dot{\theta}_{2} \\
& n_{21}=-r_{1} \cos \phi_{1}+\frac{(x+b) r_{1}}{2 b} \cos \phi_{1} \\
& n_{22}=-\frac{(x+b) r_{2}}{2 b} \cos \phi_{2} \\
& n_{23}=r_{1} \sin \phi_{1} \dot{\theta}_{1}-\frac{(x+b) r_{1}}{2 b} \sin \phi_{1} \dot{\theta}_{1} \\
& n_{24}=\frac{(x+b) r_{2}}{2 b} \sin \phi_{2} \dot{\theta}_{2}
\end{aligned}
$$




$$
\begin{aligned}
n_{31} & =\frac{r_{1}}{2 b} \cos \phi_{1} & n_{32} & =-\frac{r_{2}}{2 b} \cos \phi_{2} \\
n_{33} & =-\frac{r_{1}}{2 b} \sin \phi_{1} \dot{\theta}_{1} & n_{34} & =\frac{r_{2}}{2 b} \sin \phi_{2} \dot{\theta}_{2} \\
n_{41} & =-r_{1} \sin \phi_{1} & n_{42} & =r_{2} \sin \phi_{2} \\
n_{43} & =-r_{1} \cos \phi_{1} \dot{\theta}_{1} & n_{44} & =r_{2} \cos \phi_{2} \dot{\theta}_{2} .
\end{aligned}
$$

It should be emphasized that a necessary and sufficient condition for the matrix $N(\underline{x})$ to remain nonsingular is that the longitudinal speed $\dot{y}$ should remain positive.

The overall dynamic system model is given by

$$
\underline{\ddot{y}}=M(\underline{x})+N(\underline{x})\left[\begin{array}{c}
\ddot{\theta}_{1} \\
\ddot{\theta}_{2} \\
\dot{\phi}_{1} \\
\dot{\phi}_{2}
\end{array}\right], \quad \begin{aligned}
& \ddot{\theta}_{1}+\alpha_{1} \dot{\theta}_{1}=\beta_{1} u_{1} \\
& \ddot{\theta}_{2}+\alpha_{2} \dot{\theta}_{2}=\beta_{2} u_{2} \\
& \ddot{\phi}_{1}+\alpha_{3} \dot{\phi}_{1}=\beta_{3} u_{3} \\
& \ddot{\phi}_{2}+\alpha_{4} \dot{\phi}_{2}=\beta_{4} u_{4} .
\end{aligned}
$$

\section{NONLINEAR CONTROL STRATEGY}

The control objective in this paper is to control a sheet on the horizontal plane from an initial state with nonzero longitudinal velocity to a final state also with nonzero longitudinal velocity. The control strategy presented in this section assumes a realistic second-order model for all actuators, as derived and validated in Section V-B. The control strategy developed for this system is also dynamic and uses concepts of linearization by state feedback [23] and a backstepping technique similar to dynamic surface control [24]. The desired sheet trajectories lateral and longitudinal coordinates $x$ and $y$ for the sheet's leading edge right corner $C$, the sheet angular rotation $\phi$, and the buckle $\delta$ are given by

$$
\begin{aligned}
& x_{d}(t)=\dot{x}_{d}(t)=\ddot{x}_{d}(t)=0 \\
& y_{d}(t)=\alpha t, \quad \dot{y}_{d}(t)=\alpha, \quad \ddot{y}_{d}(t)=0 \\
& \phi_{d}(t)=\dot{\phi}_{d}(t)=\ddot{\phi}_{d}(t)=0 \\
& \delta_{d}(t)=-c_{1}, \quad \dot{\delta}_{d}(t)=\ddot{\delta}_{d}(t)=0
\end{aligned}
$$

for $t=[0, T]$, where $\alpha$ and $T$ are, respectively, the desired longitudinal velocity of the sheet and time at which the sheet must leave the steerable nips mechanism. The amount of buckle is $c_{1}$. The block diagram of the proposed control strategy is shown in Fig. 15. The control law is given by

$$
\left[\begin{array}{c}
\ddot{\theta}_{1 d} \\
\ddot{\theta}_{2 d} \\
\dot{\phi}_{1 d} \\
\dot{\phi}_{2 d}
\end{array}\right]=N(\underline{x})^{-1}\left(\left[\begin{array}{l}
\ddot{x}_{d}+\left(K_{1}+\lambda_{1}\right) \dot{\tilde{x}}+\lambda_{1} K_{1} \tilde{x} \\
\ddot{y}_{d}+\left(K_{2}+\lambda_{2}\right) \dot{\tilde{y}}+\lambda_{2} K_{2} \tilde{y} \\
\ddot{\phi}_{d}+\left(K_{3}+\lambda_{3}\right) \dot{\tilde{\phi}}+\lambda_{3} K_{3} \tilde{\phi} \\
\ddot{\delta}_{d}+\left(K_{4}+\lambda_{4}\right) \dot{\tilde{\delta}}+\lambda_{4} K_{4} \tilde{\delta}
\end{array}\right]-M(\underline{x})\right)
$$

$$
\begin{aligned}
& C_{1}(s)=\frac{k_{p 1}\left(s+\gamma_{1}\right)\left(s+\gamma_{2}\right)}{s^{2}} \quad C_{2}(s)=\frac{k_{p 2}\left(s+\gamma_{3}\right)\left(s+\gamma_{4}\right)}{s^{2}} \\
& C_{3}(s)=\frac{k_{s 1}\left(s+\eta_{1}\right)}{s\left(s+\eta_{2}\right)} \quad C_{4}(s)=\frac{k_{s 2}\left(s+\eta_{3}\right)}{s\left(s+\eta_{4}\right)}
\end{aligned}
$$

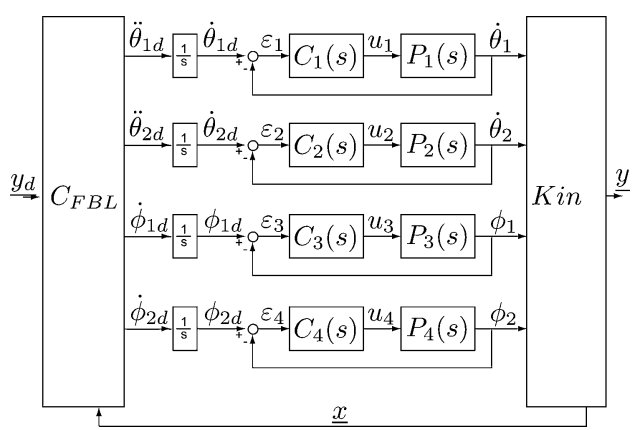

Fig. 15. Control system block diagram.

Equation (27), which is schematically depicted in Fig. 15 by the block $C_{F B L}$, linearizes through feedback the kinematics and generates the desired angular accelerations and desired steering velocities for each of the rollers. These desired values are then integrated to obtain reference trajectories, which are tracked by local inner linear controllers given by (28) and represented by the blocks $C_{i}(s), i=1,2,3$, 4 , in Fig. 15, where $\eta_{i}$ and $\gamma_{i}(i=1,2,3,4)$ are positive coefficients. The actuators' process dynamics are represented by the blocks $P_{1}(s), i=1,2$, in Fig. 15 while the steering dynamics are represented by the blocks $P_{i}(s), i=3,4$.

A formal convergence analysis of this control strategy is provided in [9], [10], and [19]. Moreover, they also provide a systematic methodology to tune the control system so that, given a specified maximum nominal longitudinal speed $\alpha$ and a minimum travel time $T=\alpha / L$, where $L$ is the length of the sheet, control gains can be determined so that the magnitude of tracking errors is guaranteed to be attenuated, from specified maximum initial values, to final values that are within specified allowable values.

In the control strategy in (27) and (28), feedback linearization is used only to linearize the kinematics of the system, while internal loops are used to locally control the actuator's positions and velocities. Through extensive simulation and experimental results, such as those presented in Section VII, we have found that this control strategy performs robustly to parameter and dynamic uncertainty in the actuators. This robustness can be attributed to the fact the feedback linearization portion of the controller does not depend on the actuator dynamics. In contrast, we have found [8] that control strategies that are based on direct output feedback linearization plus dynamic extension [23] of the overall plant given (25) do not perform well, under identical conditions, due to parameter and dynamic uncertainty in the actuators. A comprehensive robustness analysis is also presented in [8], which shows that the control strategy based on kinematic feedback linearization with actuator internal loops is significantly more robust to parameter and dynamic uncertainty in the actuators than the control strategy that is based on the direct output feedback linearization plus dynamic extension of the overall dynamics.

\section{Simulation AND EXPERIMENTAL RESUltS}

Simulated and experimental results were obtained for a sheet with initial conditions, as shown in Table III. The values for 
TABLE III

INITIAL EXPERIMENTAL AND SIMULATED SHEET ERRORS

\begin{tabular}{|c|c|c|}
\hline Variable Name & Value & Units \\
\hline Sheet Lateral Error & 5.2 & millimeters \\
Sheet Longitudinal Error & -50 & millimeters \\
Sheet Angle Position & 0.7 & degrees \\
Buckle & 0 & millimeters \\
Forward Velocity & 0.5 & meters/second \\
Available Control Time & 0.35 & seconds \\
\hline
\end{tabular}

TABLE IV

CONTROL VARIABLES

\begin{tabular}{|c|c|}
\hline Variable & Value \\
\hline$k_{p 1}$ & 0.5 \\
$k_{p 2}$ & 0.5 \\
$\gamma_{1}$ & 20.7 \\
$\gamma_{2}$ & 29.0 \\
$\gamma_{3}$ & 20.7 \\
$\gamma_{4}$ & 29.0 \\
$k_{s 1}$ & 1200 \\
$k_{s 2}$ & 1200 \\
$\eta_{1}$ & 7.6 \\
$\eta_{2}$ & 14.6 \\
$\eta_{3}$ & 7.6 \\
$\eta_{4}$ & 14.6 \\
\hline
\end{tabular}
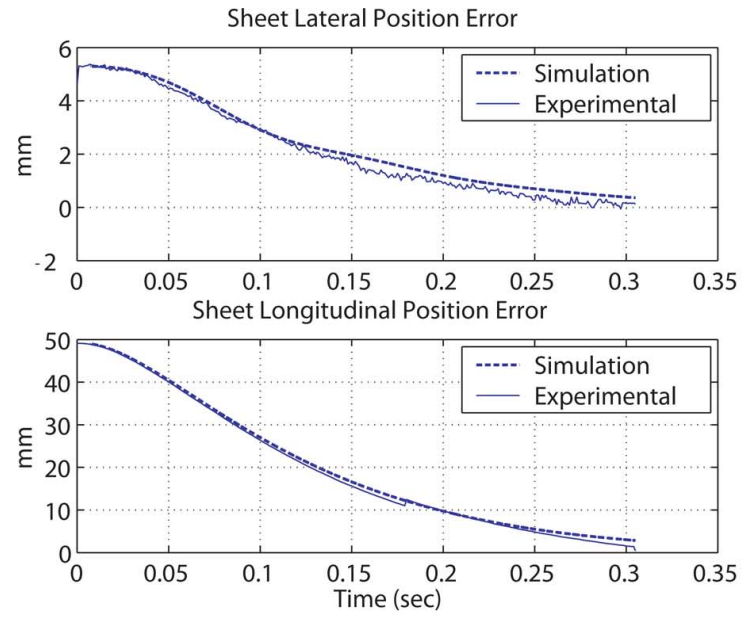

(a)
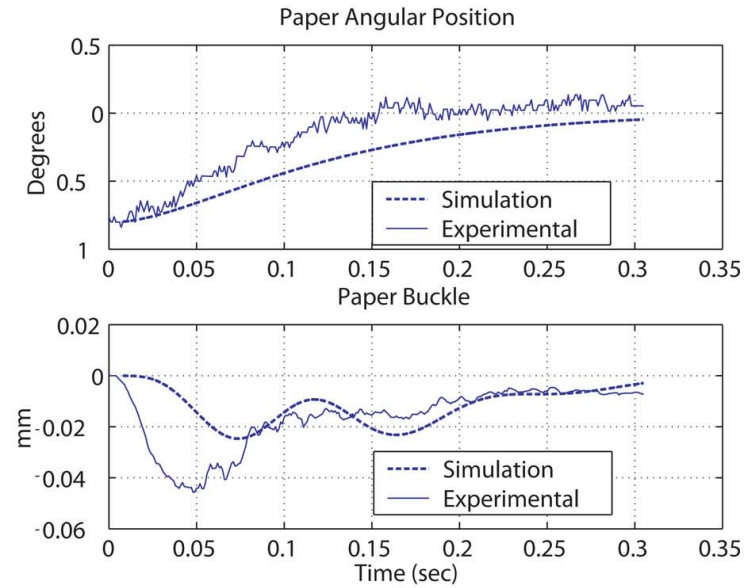

(b)

Fig. 16. (a) Sheet lateral and longitudinal errors. (b) Sheet angular position and sheet buckle.
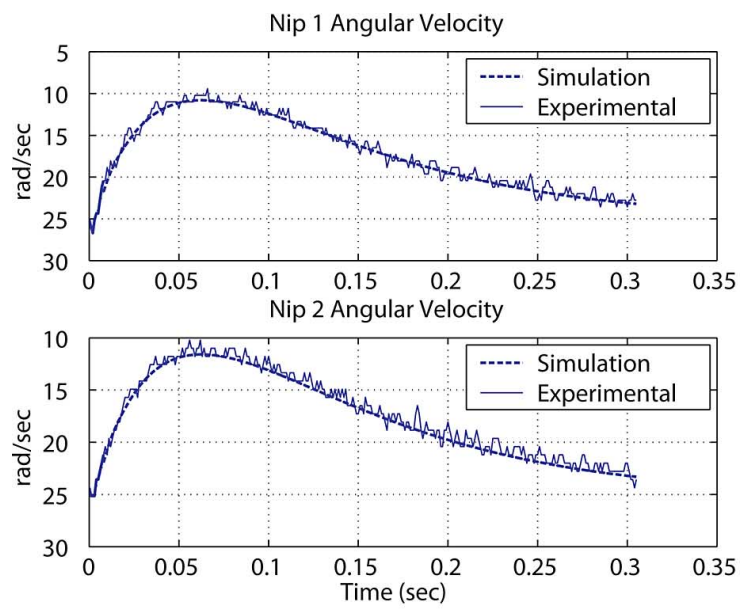

(a)
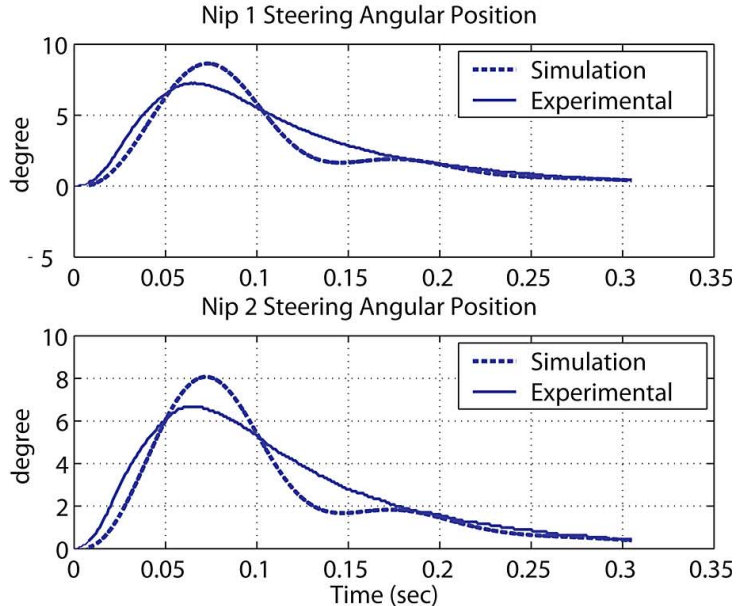

(b)

Fig. 17. (a) Roller angular velocity. (b) Roller steering angle.

the aforementioned control parameters are shown in Table IV. Each of the control gains is the combination of all system gains such as gains of the amplifier, conversion factor from radians to encoder pulses, etc. The sheet was steered from this initial condition to a desired longitudinal, lateral, and angular position. In addition, the sheet was kept with a small amount of buckle to avoid stretching the sheet. Fig. 16(a) shows the sheet longitudinal and lateral errors, and Fig. 16(b) shows the angular position and amount of paper buckle. The errors were reduced in the time allowed, less than $0.382 \mathrm{~s}$. This time is dependent on the sheet velocity, the amount of skew error, the gap between lateral sensors, and the sizes of a sheet. For example, for an A4 sheet (194.6 $\times$ $279.4 \mathrm{~mm}$ ), traveling at the velocity of $0.5 \mathrm{~m} / \mathrm{s}$ with a maximum angular error of $0.025 \mathrm{rad}$ and a sensor gap of $21.3 \mathrm{~mm}$ results in an allowable time of $0.382 \mathrm{~s}$ for correction for all errors. The final errors are within the requirements: less than $\pm 1.3 \mathrm{~mm}$ in the lateral direction, $\pm 1.6 \mathrm{~mm}$ in the process direction, and $\pm 3.5 \mathrm{mrad}$ of angular error. Experimental and simulated results of sheet errors in Fig. 16(a) and (b) show some discrepancies. These discrepancies can be attributed to sensor noise and slip between the ball and the sheet. The rollers' angular velocities and steering angular positions are shown in Fig. 17(a) and (b). 
As it was expected, the initial and final angular velocities are equivalent to the sheet's nominal longitudinal velocity once the errors have been corrected. The steering angular position of each roller is close to zero once the sheet's errors have been corrected.

Current sensing equipment limit the control precision at high speeds. Laser sensors noise level increases at high speeds decreasing the precision of sensors.

\section{CONCLUSION}

This paper presented an innovative mechanism to improve paper handling performance in a printer paper handling system, when it is placed before the image registration station. This mechanism uses steerable nips to control the motion of a sheet of paper on a horizontal plane, from the time instant when the leading edge of the sheet enters in contact with the nips until the time instant when the trailing edge of the sheet losses contact with the nips and enters the registration station, in order to reduce longitudinal, lateral, and angular position tracking errors to acceptable levels.

A prototype of this device was constructed and tested. Kinematic and dynamic models of the steerable nips mechanism were derived under the assumptions that the mass of the sheet can be neglected and that the paper sheet remains sufficiently stiff under minute levels of buckling to sustain rigid body rotation, and were validated with experimental data. A control strategy was presented in this paper comprised of a nonlinear outer feedback linearization loop and inner dynamic surface control loops around each of the actuators. The outer loop linearizes the paper sheet kinematics under the steerable nips mechanism action and generates desired rotating speeds and steering angles for each of the two steerable nips. These reference trajectories are tracked by the four inner dynamic surface control feedback loops. Simulation and experimental results are in close agreement and show that by using the proposed control strategy, the steerable nips mechanism can correct for large initial sheet tracking errors, which exceed industry specifications, and attain sheet tracking errors that are below industry requirements when the sheet enters the registration station, without damaging or marking the sheet. Small discrepancies between the experimental and simulation results were attributed to sensor noise, irregularities in the edge of the paper, and slight model parameter mismatches, particularly among the damping ratios of the actuators dynamics.

\section{REFERENCES}

[1] B. Bukkems, R. van de Molengraft, M. Heemels, N. van de Wouw, and M. Steinbuch, "A piecewise linear approach towards sheet control in a printer paper path," in Proc. Amer. Control Conf., 2006, pp. 1315-1320.

[2] G. Campion, B. d'Andrea Novel, and G. Bastin, "Controllability and state feedback stabilisability of nonholonomic mechanical systems," in Proc. Adv. Robot Control: Proc. Int. Workshop Nonlinear Adaptive Control: Issues Robot., Grenoble, France, Nov. 1990, pp. 106-124.

[3] C. Cloet, M. Kruciński, R. Horowitz, and M. Tomizuka, "A hybrid control scheme for a copier paperpath," in Proc. 1999 Amer. Control Conf., San Diego, CA, Jun., pp. 2114-2118.

[4] C. Cloet, M. Kruciński, R. Horowitz, and M. Tomizuka, "Intersheet spacing control and controllability of a copier paperpath," in Proc. 1998 IEEE Conf. Control Appl., Trieste, Italy, Sep., pp. 726-730.
[5] C. Cloet, M. Tomizuka, and R. Horowitz, "Design requirements and reference trajectory generation for a copier paperpath," presented at the Conf. Adv. Intell. Mechatronics, Como, Italy, Jul. 2001.

[6] C. Clote, "A mechatronics approach to copier paperpath design," Ph.D dissertation, Univ. California, Berkeley, 2001.

[7] J. G. Elliot and R. F. Gans, "Closed-loop control of an underactuated sheet registration device using feedback linearization and gain scheduling," IEEE Trans. Control Syst. Technol., vol. 16, no. 4, pp. 589-599, Jul. 2008

[8] E. Ergueta, R. Seifried, and R. Horowitz, "A robust approach to dynamic feedback linearization for steerable nips mechanism," presented at the 2008 ASME Dyn. Syst. Control Conf., Ann Arbor, MI, Oct. 2008.

[9] E. Ergueta, R. Sanchez, R. Horowitz, and M. Tomizuka, "Convergence analysis of a steerable nips mechanism for full sheet control in printing devices," ASME J. Dyn. Syst. Meas. Control, 2007, to be published.

[10] E. Ergueta, R. Sanchez, R. Horowitz, and M. Tomizuka, "Full sheet control through the use of steer-able nips," presented at the 2007 ASME IMECE, Seattle, WA, Nov. 2007, Paper IMECE2007-41279.

[11] N. Y. Hwang and S. S. Penfield, "Sheet registration and deskewing system with independent drives and steering," U.S. Patent 6634 521, Oct. 2003.

[12] J. J. T. H. de Best, B. H. M. Bukkems, M. J. G. van de Molengraft, W. P. M. H. Heemels, and M. Steinbuch, "Robust control of piecewise linear systems: A case study in sheet flow control," Control Eng. Pract., vol. 16, no. 8, pp. 991-1003, Aug. 2008.

[13] M. Krucinski, "Feedback control of photocopying machinery," Ph.D. dissertation, Univ. California, Berkeley, 2000.

[14] M. Krucinski, C. Cloet, R. Horowitz, and M. Tomizuka, "Asynchronous observer for a copier paperpath," in Proc. 37th IEEE Conf. Decis. Control, Dec. 1998, vol. 3, pp. 2611-2612.

[15] M. Kruciński, C. Cloet, R. Horowitz, and M. Tomizuka, "Interobject spacing control and controllability of a manufacturing transportation system," in Proc. 1999 Amer. Control Conf., Jun., vol. 2, pp. 1259-1265, Session WA03-4.

[16] M. Kruciński, C. Cloet, R. Horowitz, and M. Tomizuka, "A mechatronics approach to copier paperpath control," presented at the 1st IFAC Conf. Mechatronics Syst., Darmstadt, Germany, Sep. 2000.

[17] R. M. Murray, Z. Li, and S. S. Sastry, A Mathematical Introduction to Robotic Manipulation. Boca Raton, FL: CRC Press, 1993.

[18] S. Rai and W. Jackson, "A hybrid hierarchical control architecture for paper transport systems," in Proc. 37th IEEE Conf. Decis. Control, Tampa, FL, Dec. 1998, pp. 4294-4295.

[19] R. Sanchez, "Nonlinear control strategies for a steerable nips mechanism," Ph.D. dissertation, Univ. California, Berkeley, 2006.

[20] R. Sanchez, E. Ergueta, B. Fine, R. Horowitz, M. Tomizuka, and M. Krucinski, "A mechatronic approach to full sheet control using steerable nips," presented at the 4th IFAC-Symp. Mechatron. Syst., Heidelberg, Germany, Sep. 2006.

[21] R. Sanchez, R. Horowitz, and M. Tomizuka, "Paper sheet control using steerable nips," in Proc. 2004 Amer. Control Conf., Boston, MA, Jun. 30-Jul. 2, pp. 482-487.

[22] R. Sanchez, R. Horowitz, M. Tomizuka, and S. N. Simić, "Full paper sheet control using hybrid automata," in Proc. 2004 Hybrid Syst.: Comput. Control, Philadelphia, PA, Mar., pp. 523-538.

[23] S. S. Sastry, Nonlinear Systems : Analysis, Stability, and Control. New York: Springer-Verlag, 1999.

[24] D. Swaroop, J. C. Gerdes, P. P. Yip, and J. K. Hedrick, "Dynamic surface control of nonlinear systems," in Proc. Amer. Control Conf., Albuquerque, NM, Jun.1997, vol. 5, pp. 3028-3034.

[25] N. Tanaka, H. Fukumoto, K. Arimoto, and Y. Iwashita, "Skew correction mechanism for thermal transfer type color printers," in Proc. Int. Conf. Micromechatronics Inf. Precis. Equipment, Tokyo, Japan, Jul. 1997, pp. 635-638.

[26] P. Tunestål and M. Kruciński, "Hybrid control of a manufacturing transport system," in Proc. 1997 IEEE Conf. Decis. Control, San Diego, CA, Dec. 1997, pp. 84-89, Session ID WA03-4.

[27] D. Wang and G. Xu, "Full-state tracking and internal dynamics of nonholonomic wheeled mobile robots," IEEE/ASME Trans. Mechatronics, vol. 8, no. 2, pp. 203-214, Jun. 2003.

[28] Y. Xu and S. K.-W. Au, "Stabilization and path following of a single wheel robot," IEEE/ASME Trans. Mechatronics, vol. 9, no. 2, pp. 407-419, Jun. 2004.

[29] X. Yun and N. Sarkar, "Dynamic feedback control of vehicles with two steerable wheels," in Proc. 1996 IEEE Int. Conf. Robot. Autom., 1996, pp. 3105-3110. 


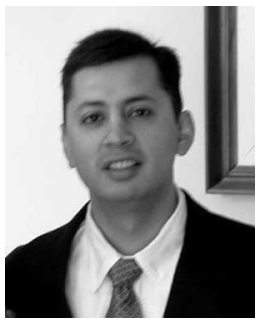

Rene Sanchez (M'09) was born in Gualaceo, Ecuador, in 1970. He received the B.S. and M.S. degrees in mechanical engineering from Rensselaer Polytechnic Institute, Troy, NY, in 1993 and 1995, respectively, and the Ph.D. degree from the University of California, Berkeley, in 2006.

From 1995 to 2000 , he was with the Xerox Research Center. Since 2006, he has been with Nikon Research Corporation of America, Belmont, CA. His current research interests include nonlinear, learning, motion, and adaptive control related to robotics, precision movement, and manufacturing.

Dr. Sanchez is a member of the American Society of Mechanical Engineers (ASME).

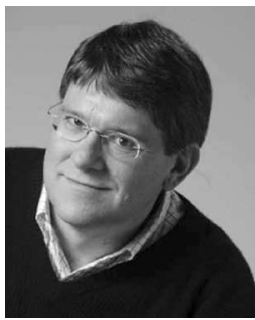

Roberto Horowitz (M'89-SM'02) was born in Caracas, Venezuela, in 1955. He received the B.S. degree (with highest honors) and the Ph.D. degree in mechanical engineering from the University of California, Berkeley, in 1978 and 1983, respectively.

In 1982, he joined the Department of Mechanical Engineering, University of California, Berkeley, where he is currently a Professor. His current research interests include the areas of adaptive, learning, nonlinear, and optimal control, with applications to microelectromechanical systems (MEMS), computer disk file systems, robotics, mechatronics, and intelligent vehicle and highway systems (IVHSs).

Prof. Horowitz is a member of the American Society of Mechanical Engineers (ASME).

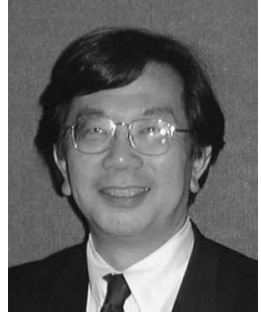

Masayoshi Tomizuka (M'86-SM'95-F'97) was born in Tokyo, Japan, in 1946. He received the B.S and M.S. degrees in mechanical engineering from Keio University, Tokyo, and the Ph.D. degree in mechanical engineering from Massachusetts Institute of Technology, Cambridge, in 1974.

Since 1974 , he has been with the University of California, Berkeley, where he is currently the Cheryl and John Neerhout, Jr., Distinguished Professorship Chair. He teaches courses in dynamic systems and controls. His current research interests include optimal and adaptive control, digital control, signal processing, motion control, and control problems related to robotics, machining, manufacturing, information storage devices, and vehicles. He was a consultant to various organizations, including General Electric, General Motors, and United Technologies.

Dr. Tomizuka was the Editor-in-Chief of the IEEE/ASME TRANSACTIONS ON MECHATRONICS from 1997 to 1999. From 1998 to 1999, he was the President of the American Automatic Control Council (AACC). From 2002 to 2004, he was the Program Director of the Dynamic Systems and Control Program in the Civil and Mechanical Systems Division, National Science Foundation. $\mathrm{He}$ is a Fellow of the ASME and the Society of Manufacturing Engineers. He received the Charles Russ Richards Memorial Award from ASME in 1997, the Rufus Oldenburger Medal from ASME in 2002, and the John R. Raggazini Award from AACC in 2006. From 1988 to 1993, he was the Technical Editor of the American Society of Mechanical Engineers (ASME) Journal of Dynamic Systems, Measurement and Control (J-DSMC). 